\title{
Devices Selection and Topology Comparison of Medium Voltage DC Solid State Circuit Breakers
}

\author{
Yihui Zhang \\ Electrical Engineering Department, North China Electric Power University, Beijing 102206, China
}

\begin{abstract}
DC grid has been treated as a viable solution to solve green-house effect and reduce the cost of fossil fuels. Compared with AC grid, DC grid shows many inherent advantages, such as high efficiency power delivery, less expensive to deploy and no need for phase and frequency synchronization. However, over current protection for $\mathrm{DC}$ grid require fast response. To solve this problem, solid state DC circuit breakers need to be promoted. In this thesis, thermal property was taken into consideration to find the most suitable semiconductor devices. Per unitized thermal resistance of the heat sink to the ambient was used to find the reasonable conducting current. Module MOV topology and one MOV topology were compared to see the voltage and current stress.
\end{abstract}

Keywords-solid staate circuit breakers; medium voltage; $\mathrm{MOV}$

\section{INTRODUCTION}

With the rapid development of power system, modern power system is facing a series of new contradictions and problems, but it is also faced with unprecedented challenges. With the power system continuing to expand, the short circuit current level is rapidly increased. Because of the slow functioning speed, the difficulty of arc extinguishes, and the limitation of the capacity, the existing circuit breakers cannot meet the requirement of the increasing breaking capacity. In many situations, functioning speed is significant, especially about the power system stability control. For that reason, the way to limit and interrupt the fault current rapidly becomes more and more important. However, the functioning speed of traditional mechanical circuit breakers were limited by the mechanical inertia, this hindered the improvement of the functioning speed of mechanical circuit breakers severely, and impact the fault interruption and the stability of the grid control. In addition, the reliability and the life of mechanical circuit breakers also cannot meet the requirement of modern power system.

In high voltage and medium voltage field, there are only high voltage DC circuit breaker which can transfer or breaking load current of the high voltage DC circuit breaker ${ }^{[1]}$. There are some companies did the research of the circuit breakers which can interrupt short circuit fault current. The DC solid state circuit breakers are developed with the technique of power electronic devices. In 1990s, a solid state switches with thyristors appeared. After that, the appearing of fully controllable devices such as integrated gate commutated thyristor (IGCT), insulated gate bipolar transistor (IGBT), etc makes further development of DC solid state circuit breakers. At present, solid-state DC circuit breakers based on power electronic devices can be divided into hybrid DC circuit breakers and solid-state DC circuit breakers ${ }^{[2]}$. The hybrid DC circuit breaker consists of power electronic devices and mechanical switches in parallel. Although under the normal operate condition, there is almost no on state loss, the time delay of interrupting the fault current is still determined by the mechanical circuit breaker action time. For all solid-state DC circuit breakers, although breaking time is short, there are high expense, high on-state loss, limited capacity of single device and other shortcomings. The continuous development of semiconductor devices, the reduction of devices losses, and the improvement of capacity provide a good foundation for the development of DC solid state circuit breakers.

This paper is aimed to develop medium voltage DC solid circuit breakers. Based on the existing power electronic devices. It is necessary to series and parallel semiconductors to achieve medium voltage DC solid circuit breakers.

\section{DEVICE SELECTION}

This paper is aimed at a medium voltage DC solid state circuit breakers, one thing need to be taken into consideration is its on state loss. For the devices need to be selected, the nominal voltage of each device is kilo volts level, and has an on-state voltage drop in the order of some volts. This means constant losses in the kilowatt range when conducting some hundred amperes. Every device has a nominal operation temperature, usually the maximum temperature is no more than $150{ }^{\circ} \mathrm{C}$. Because the resistance is proportional to the temperature, to achieve lower on state loss, it is important to keep the devices under a reasonable temperature. What is more, when the short circuit fault occurs, the switches need to interrupt the fault current. Due to its large value, there must be a large amount of heat generated by the semiconductors, so it is very necessary to take into thermal properties into consideration to keep safe and efficiency operation. A violation of the temperature ratings can lead to a reduced safe operating area and consequently a sudden device failure or to a reduced operational lifetime. If the thermal property of the semiconductors can hardly meet the requirement, this will require a large and complex cooling system.

The value for RT resistance can be easily calculated from the IGBT output characteristic curve from the datasheet. From Figure 1 we can linear the output characteristic curve around a very little fraction of the curve to get the resistance value. VTO is the threshold voltage which can get from the datasheet. For MOSFET, the value of VTO is zero. 
The average conduction loss by IGBT is calculated by the following equation:

$$
\begin{gathered}
V_{C E s a t}=\mathrm{V}_{\mathrm{TO}}+R_{T} \times I_{C} \\
P_{\text {cond }}=V_{C E s a t} \times I_{c} \\
R_{T}=\frac{\Delta V}{\Delta I}=\frac{V_{C E 2}-V_{C E 1}}{\mathrm{I}_{C 2}-\mathrm{I}_{C 1}}
\end{gathered}
$$

After we get the value of conduction loss, we can start calculating the value of the thermal resistance between the heat sink and the ambient.The value of the thermal resistance Rsa can be easily get from the following equation:

$$
\frac{T_{j \max }-T_{A}}{P_{\text {cond }}}=R_{S A}+R_{J C}+R_{C S}
$$

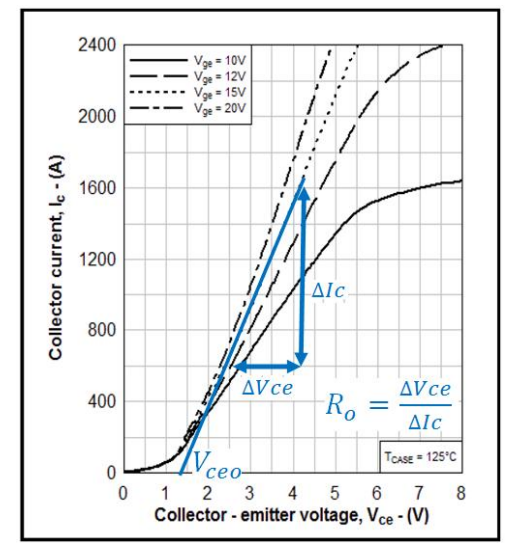

FIGURE I. IGBT OUTPUT CHARACTERISTIC

\section{A. Thermal Resistance}

The value of Tjmax, Rjc and Rcs can be got from the date sheet, and Pcond can be calculated from equation (2). After we get the value of RSA we should per unitize it to check the thermal property of this condition. As the thermal resistance is inversely proportional to area, to find the thermal resistance of a certain area cold plate, we should multiply the area (in square inch) of the cold plate.

The purpose of calculating the RSA is that it is a value to show whether the certain conduction loss caused by a certain value of a current can meet the requirement of the thermal property. When the conducting current is so big, it will cause the cooling system cannot cool the heat through the device. around $0.1-0.2$ in per unitize. The lower the value the higher requirement of the cold plate. If the value of RSA is lower than performance of the semiconductors and reduce the lifetime of devices.This will lead to devices overheated which may damage the From [3] we can know that the reasonable value of RSA is 0.1 this means the big pressure for the cold plate. This will cause the cold plate thicker of the cooling water runs much faster, which is not reality. So when the value of $\mathrm{R}_{\mathrm{SA}}$ smaller than 0.1 , Ic should be decreased. The existing cooling system The existing cooling system cannot meet the thermal requirement or cool the heat which generated by that current value. The strategy of finding the proper conducting current value is:If it is less than 0.1 , reduce the current valueIf it is greater than 0.1 , increase the current value

\section{B. Efficiency Calculation}

From the literature ${ }^{[4]}$ we know that parallel semiconductors can increase the efficiency of the circuit breakers, so after meet the requirement of the thermal requirement, the efficiency of the devices also need to be taken into consideration. Because the rating system capacity is $40 \mathrm{MW}$, the efficiency should be high enough to reduce the conduction loss. The efficiency should greater than 99.99 , even that the value of the conduction loss is still not a little number. In order to achieve that, we can lower the current of every single semiconductor and parallel more modules. The efficiency of the circuit breaker is given as,

$$
\text { Efficiency }=\frac{40 \mathrm{MW}}{\text { total loss }+40 \mathrm{MW}}
$$

\section{Device Selection}

After the basic knowledge of thermal property and the efficiency calculation we can use the methods above to select proper devices. Things need to be taken into consideration when select semiconductor for medium voltage DC solid state circuit breakers are rating voltage, rating current, thermal properties, efficiency and the number of the devices.Table 2 is the devices we choose from.

TABLE I. SYSTEM SPECIFICATION

\begin{tabular}{|c|c|}
\hline ratings & values \\
\hline Voltage rating & $20 \mathrm{kV}$ \\
\hline Current rating & $2000 \mathrm{~A}$ \\
\hline System capacity rating & $40 \mathrm{MVA}$ \\
\hline
\end{tabular}

TABLE II. COMPONENT LIST

\begin{tabular}{|l|l|}
\hline Manufacturer & Specifications \\
\hline Infineon IGBT $^{[5]}$ & $6.5 \mathrm{kV} \mathrm{600A}$ \\
\hline Infineon IGBT $^{[6]}$ & $6.5 \mathrm{kV} 750 \mathrm{~A}$ \\
\hline ABB IGBT $^{[7]}$ & $6.5 \mathrm{kV} 600 \mathrm{~A}$ \\
\hline ABB IGBT $^{[8]}$ & $6.5 \mathrm{kV} 750 \mathrm{~A}$ \\
\hline ABB GTO $^{[9]}$ & $4.5 \mathrm{kV} 210 \mathrm{~A}$ \\
\hline ABB Thyristor $^{[10]}$ & $3 \mathrm{kV}$ \\
\hline ABB Thyristor $^{[1]}$ & $2.4 \mathrm{kV}$ \\
\hline
\end{tabular}

Table 3 is the result of the thermal property calculation.

According to the calculation result we can see that IGBT is the most suitable devices considering the thermal property and the number of the semiconductor. After thermal calculation we need to do the efficiency calculation, and the efficiency should greater than 99.99. In order to achieve that, we can lower the current of every single IGBT and parallel more modules.

After calculation, Infineon $6.5 \mathrm{kV} 750 \mathrm{~A}$ is the most suitable device. The number of device is 6 modules in series, 2 
branches in parallel, which makes its total number 12.

TABLE III. CALCULATION RESULT

\begin{tabular}{|c|c|c|c|c|c|}
\hline $\begin{array}{c}\text { Parameters } \\
\text { Types }\end{array}$ & RsaK/kW & $\begin{array}{c}\text { p.u./K/W } \\
\text {.inch }\end{array}$ & series & parallel & total \\
\hline $\begin{array}{c}\text { Infineon IGBT } \\
6.5 \mathrm{kV} \text { 600A }\end{array}$ & 13.3746 & 0.17 & 6 & 4 & 12 \\
\hline $\begin{array}{c}\text { Infineon IGBT } \\
\text { 6.5kV 750A }\end{array}$ & 9.23258 & 0.16 & 6 & 3 & 6 \\
\hline $\begin{array}{c}\text { ABB IGBT } \\
\text { 6.5kV 600A }\end{array}$ & 7.01725 & 0.14 & 6 & 5 & 12 \\
\hline $\begin{array}{c}\text { ABB IGBT } \\
\text { 6.5kV 750A }\end{array}$ & 6.031746 & 0.12 & 6 & 4 & 12 \\
\hline $\begin{array}{c}\text { ABB Thyristor } \\
\text { 2.4kV }\end{array}$ & 29.39203 & 0.12 & 9 & 2 & 18 \\
\hline $\begin{array}{c}\text { ABB Thyristor } \\
\text { 3kV }\end{array}$ & 16.91786 & 0.11 & 7 & 3 & 21 \\
\hline $\begin{array}{c}\text { ABB Thyristor } \\
\text { 3kV }\end{array}$ & 15.49669 & 0.10 & 7 & 3 & 21 \\
\hline $\begin{array}{c}\text { ABB GTO } \\
4.5 \mathrm{kV} 210 \mathrm{~A}\end{array}$ & 44.70256 & 0.20 & 5 & 10 & 50 \\
\hline
\end{tabular}

III. SimUlATION AND RESUltS

The software used in this simulation is ANSYS Simplorer. ANSYS Simplorer is a powerful platform for modeling, simulating and analyzing system-level digital prototypes. It has a unique ability to integrate high-fidelity models of power electronics.

The module MOV topology is shown as Figure2. Each paralleled module was protected by a MOV. As there are six modules in series together, there are six MOVs protect these modules separately. The one MOV module only has a MOV to protect the whole modules, shown as Figure 3. These two different topologies may have different voltage stress and current stress. Analysis will be taken to compare the characteristic of these two topologies.

\section{A. Ideal Condition}

Fig 4 is module MOV mode faults occurs at $10 \mathrm{~m}$. The red line is the current curve of an IGBT module, the blue line is the voltage curve of an IGBT module and the purple line is the voltage curve of MOV. We can know from the current curve that at the beginning the circuit is under normal operation state. The current value is at the rating current value. It is $1000 \mathrm{~A}$. At $1 \mathrm{~ms}$, the system occurs a short circuits fault, the current starts to increase. The peak of the red curve is the maximum value of the fault current, and from then on, the switches start to turn off. After that the current starts to go down with the MOV dissipates the energy. Finally, the fault current turns to zero. We can know from the voltage curve of the IGBT module. First during the normal operation condition, the voltage value is only the conduction voltage drop, it is about several volts. At $1.02 \mathrm{~ms}$, that is $0.02 \mathrm{~ms}$ after the fault occurs, the circuit breakers starts to turn off. Because the switches will not turn off instantaneously, so the voltage of the IGBT module will increase gradually. We all noticed that there is a voltage spike, the reason why there is a spike is that because the existing of stray inductance between MOV and IGBT modules. During that time the current changes rapidly, so the current change rate is really high which will generate a voltage. The voltage the IGBT needs to hold are the sum of the voltage which caused by the stray inductance, and the voltage which the MOV clamps. After a short time, the current change rate goes to zero, and the voltage spike disappears. After that the voltage between the

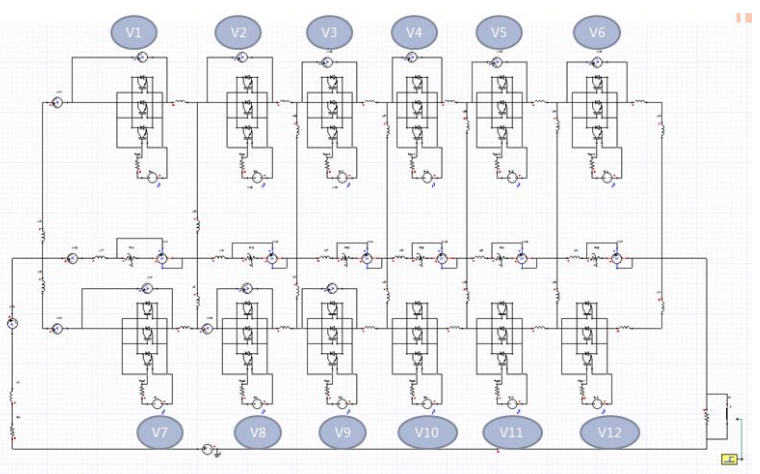

FIGURE II. MODULE MOV MODE SIMULATION MODEL

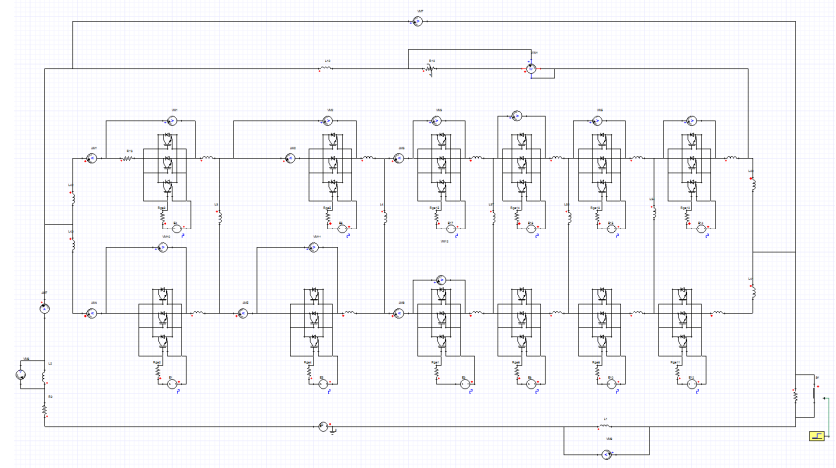

FIGURE III. ONE MOV MODE SIMULATION MODEL

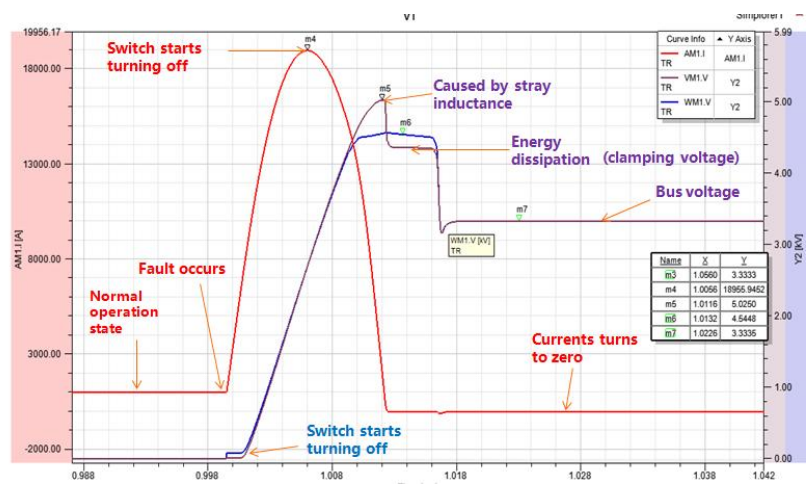

FIGURE IV. THE CURRENT AND VOLTAGE OF AN IGBT MODULE AND THE VOLTAGE OF MOV UNDER MODULE MOV MODE FAULTS OCCURS AT 10M

IGBT is the voltage which the MOV clamps, after the energy dissipation of MOV the voltage goes back to the bus voltage. We can know from the voltage curve of the MOV, when there is no fault, MOV can be treated as a resistor which has a very large resistance value. When short circuit fault occurs and the IGBT stars to turn off, that is the voltage between MOV or IGBT starts to goes up, then the resistance value of MOV decreases rapidly and can be treated as a resistor which has a 
very small resistance value. Only in this way can MOV protect the IGBT. We can also notice that the voltage value of MOV is greater than the voltage between IGBT, this is because of the parallel and series stray inductance between IGBT and MOV.

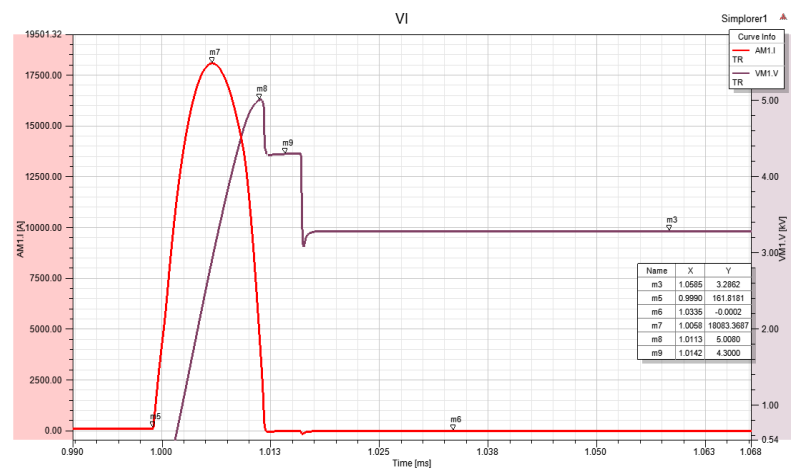

FIGURE V. FIGURE 5 THE CURRENT AND VOLTAGE OF AN IGBT MODULE AND THE VOLTAGE OF MOV UNDER ONE MOV MODE FAULTS OCCURS AT 10M

TABLE IV. DIFFERENT MODES COMPARISON

\begin{tabular}{|c|c|c|}
\hline mode & $\begin{array}{c}\text { module MOV } \\
\text { mode }\end{array}$ & one MOV mode \\
\hline Comparison index & 4.8 & 4.85 \\
\hline voltage stress/kV & 3800 & 3816 \\
\hline current stress/A & 4350 & 4375 \\
\hline energy dissipation/W & & \\
\hline
\end{tabular}

Under ideal condition, two modes are almost the same

\section{B. Non-ideal Condition}

In the most of the time, the operation condition is not ideal. It may cause the voltage sharing and current sharing problems. In this part, four non-ideal conditions were simulated to simulate the factors which would cause current and voltage problems. From Fig 6 and Fig 7, the parallel module no matter which drive signal is earlier than others no matter which branch turns off first, may need to hold a higher voltage. Compared with Fig 6 and Fig 7, the one module mode have severe voltage stress.

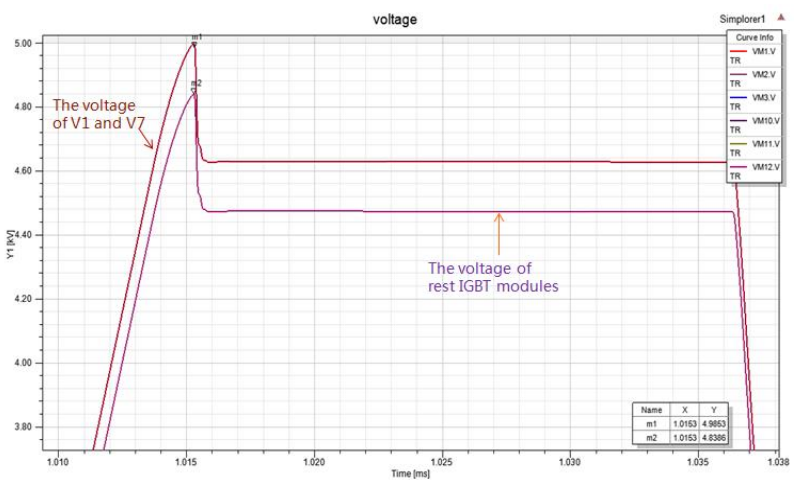

FIGURE VI. THE GATE SIGNAL OF V1 IS 2000NS EARLIER THAN OTHERS-VOLTAGE COMPARISON OF ONE MOV MODE

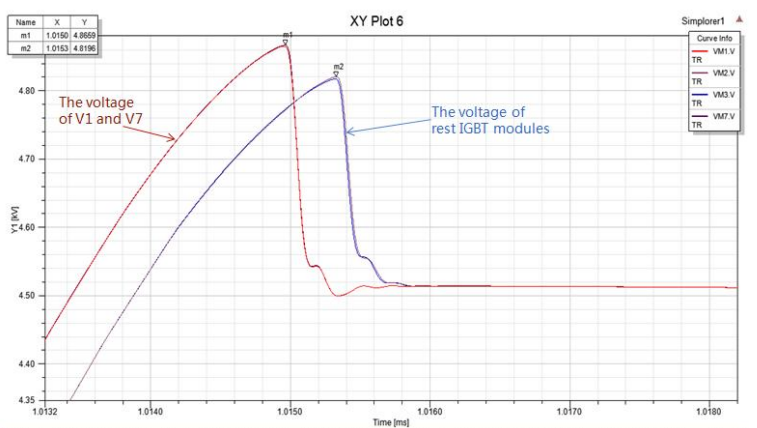

FIGURE VII. THE GATE SIGNAL OF V1 IS 2000NS EARLIER THAN OTHERS-VOLTAGE COMPARISON OF MODULE MOV MODE

\section{CONCLUSION}

In this paper, the thermal model of IGBT and diode has been compared. Then the thermal property of different kinds of semiconductor is analyzed to find the most suitable device for the medium voltage DC SSCB and to find the maximum conduction current of the devices as well. A $6.5 \mathrm{kV}, 750 \mathrm{~A}$ IGBT module from Infineon has been chosen the module MOV mode is better than one MOV mode in achieving less voltage stress of semiconductors. One MOV mode suffers more severe unbalance voltage sharing.

\section{REFERENCE}

[1] Madan, M. K., \& Chowdhury, B. H. (2012, July). Solid state fault interruption devices in medium voltage microgrid distribution systems with distributed energy resources. In Power and Energy Society General Meeting, 2012 IEEE (pp. 1-6). IEEE

[2] Shen, Z. J., Sabui, G., Miao, Z., \& Shuai, Z. (2015). Wide-bandgap solid-state circuit breakers for DC power systems: Device and circuit considerations. IEEE Transactions on Electron Devices, 62(2), 294-300.

[3] LYTRON. Product Datasheet [Online]. Available: http://www.lytron.com/Tools-and-Technical-Reference/ApplicationNotes/Selecting-A-Cold-Plate-Technology.

[4] Birk, J., \& Andresen, B. (2007, September). Parallel-connected converters for optimizing efficiency, reliability and grid harmonics in a wind turbine. In Power Electronics and Applications, 2007 European Conference on (pp. 1-7). IEEE.

[5] Infineon. Product Datasheet [Online]. Available: http://www.infineon.com/dgdl/Infineon-FZ600R65KE3-DS-v03_00EN.pdf?fileId=db3a30432c64a60d012cbbecaa6f0356

[6] Infineon. Product Datasheet [Online]. Available: http://www.infineon.com/dgdl/Infineon-FZ750R65KE3-DS-v03_01EN.pdf?fileId=db3a304325afd6e00126461 fd3936974.

[7] ABB Switzerland Ltd. Product Datasheet [Online]. Available: https://library.e.abb.com/public/d2906e423b0244b6b3566e4b72f30d39/ 5SNA\%200600G650100_5SYA\%201558-05\%2011-2015.pdf.

[8] ABB Switzerland Ltd. Product Datasheet [Online]. Available: https://library.e.abb.com/public/b47d6dffcf024d33a38f4f2bb2ed1896/5S NA\%200750G650300_5SYA\%201600-04\%2003-2016.pdf.

[9] ABB Switzerland Ltd. Product Datasheet [Online]. Available: https://library.e.abb.com/public/500afc0e0e6f00dcc125776200506d71/5 STF_11F3010.pdf.

[10] ABB Switzerland Ltd. Product Datasheet [Online]. Available: https://library.e.abb.com/public/2bc16f97ef5ee0d5c1257913004c60eb/5 STF_06T2440.pdf.

[11] ABB Switzerland Ltd. Product Datasheet [Online]. Available: https://library.e.abb.com/public/8d6eb6e4faf52ba6c1257641002a1019/5S GA\%2006D4502_5SYA1236-00.pdf 